\title{
Development and validation of risk prediction nomogram for pancreatic fistula and risk-stratified strategy for drainage management after pancreaticoduodenectomy
}

\author{
Jie Yin ${ }^{1 \#}$, Qicong Zhu ${ }^{2 \#}$, Kai Zhang ${ }^{1}$, Wentao Gao ${ }^{1}$, Junli Wu ${ }^{1}$, Zipeng Lu ${ }^{1}$, Kuirong Jiang ${ }^{1}$, Yi Miao ${ }^{1}$ \\ ${ }^{1}$ Pancreas Center, The First Affiliated Hospital with Nanjing Medical University, Nanjing, China; ${ }^{2}$ Department of Gastrointestinal and Pancreatic \\ Surgery, Zhejiang Provincial People's Hospital, People's Hospital of Hangzhou Medical College, Hangzhou, China \\ Contributions: (I) Conception and design: J Yin, Q Zhu, Z Lu; (II) Administrative support: K Jiang, Y Miao; (III) Provision of study materials or \\ patients: J Yin, Q Zhu; (IV) Collection and assembly of data: J Yin, Q Zhu; (V) Data analysis and interpretation: K Zhang; (VI) Manuscript writing: \\ All authors; (VII) Final approval of manuscript: All authors. \\ \#These authors contributed equally to this work. \\ Correspondence to: Zipeng Lu; Kuirong Jiang; Yi Miao. Pancreas Center, Department of General Surgery, The First Affiliated Hospital with Nanjing \\ Medical University, 300 Guangzhou Road, Nanjing 210029, China. Email: surgeonmark@hotmail.com; jiangkuirong@njmu.edu.cn; \\ miaoyi@njmu.edu.cn.
}

Background: Postoperative pancreatic fistula (POPF) is the major complication following pancreaticoduodenectomy (PD). We sought to develop and validate a risk prediction model for POPF after $\mathrm{PD}$ with the aim of determining personal risk probability and proposing a novel strategy for intraoperative placement and/or early-removal of prophylactic drainage.

Methods: Data from 993 patients undergoing PD from January 2012 to December 2016 were retrospectively analyzed. Patients were randomly assigned to either training cohort or validation cohort. A nomogram was formulated based on the results from multivariable regression model for prediction of POPF. Internal and external validation were carried out with calibration plot respectively.

Results: POPF occurred in $162(16.3 \%)$ patients. The final pre-/intra-operative prediction model included alanine transaminase level [odds ratio (OR) 1.00, 95\% confidence interval (CI): 1.00-1.00, P=0.023], combined portal-superior mesenteric vein resection (OR 0.22, 95\% CI: 0.05-0.95, P=0.043), pancreatic duct diameter (OR 1.48, 95\% CI: 1.11-1.96, P=0.007), intraoperative colloid infusion (OR 1.00, 95\% CI: 1.00 1.00, $\mathrm{P}=0.001$ ), pathology (OR 1.71, 95\% CI: 1.09-2.66, P=0.018). The area under the curve (AUC) was 0.667 in the training cohort and 0.621 in the validation cohort. The final postoperative prediction model included pancreatic duct diameter (OR 1.58, 95\% CI: 1.14-2.19, $\mathrm{P}=0.006$ ), intraoperative colloid infusion (OR 2.52, 95\% CI: 1.26-5.06, $\mathrm{P}=0.009)$, drainage fluid amylase on postoperative day 3 (POD3) (OR 4.70, 95\% CI: 3.30-6.70, $\mathrm{P}<0.001$ ), and neutrophil count on POD3 (OR 2.83, 95\% CI: 1.63-4.93, $\mathrm{P}<0.001$ ). The AUC was 0.809 in the training cohort and 0.797 in the validation cohort. Based on these variables, two nomogram prediction models were developed respectively. The calibration plot of the two models showed a good correlation between the expected risk and the actual risk in the low-risk range. Our risk-stratified strategy for drain management according to nomograms may be beneficial for $34.5 \%$ of patients.

Conclusions: Our study formulated and validated two nomogram models for predicting POPF that performed particularly well in the low-risk range. This tool may allow surgeons to propose a risk stratified strategy for intraoperative drain placement and early drain removal, which may be beneficial for a substantial proportion of patients.

Keywords: Pancreatic fistula; pancreaticoduodenectomy (PD); nomogram; drain management

^ ORCID: 0000-0003-1542-8264. 
Submitted Aug 12, 2021. Accepted for publication Dec 18, 2021.

doi: 10.21037 /gs-21-550

View this article at: https://dx.doi.org/10.21037/gs-21-550

\section{Introduction}

Pancreaticoduodenectomy (PD) is a complex procedure that carries a significant risk of mortality and morbidity. Recent advances in technique and perioperative management have reduced mortality after PD to $3 \%$ in high-volume centers $(1,2)$. However, the morbidity rate remains high. Postoperative pancreatic fistula (POPF) is the most common complication following PD (3-6). Various strategies have been proposed to help predict the development of POPF, guide surgeons on selecting when to place prophylactic drains, and when to consider early drain removal (7-10).

Several models for POPF risk prediction after PD have been reported, along with various drain management strategies. The Fistula Risk Score (FRS), introduced by Callery and colleagues, has been found to be a reliable intraoperative predictor of POPF (11). Using the FRS and drain fluid amylase (DFA) on postoperative day 1 (POD1), Vollmer and colleagues developed protocols for selective drainage and early drain removal, which demonstrated that drains can be safely omitted for one-quarter of PDs and removed early for nearly one half of cases $(7,12)$. However, whether FRS or alternative FRS prediction models are well validated. The investigators focused more on pre-/ intra-operative factors and neglect postoperative factors such as DFA and inflammatory marks. Meanwhile, drain management protocols based on different prediction models of POPF were not unified even in the same center. Moreover, the external validity of prediction models can be affected by institutional practice and resources, even fewer have been found to be reproducible $(11,13,14)$.

A nomogram, displaying as a simple graphical tool, combines multiple predictors by assigning relative weights to each predictor, and provides a quantitative estimate of the probability of incidence rate for individuals $(15,16)$. Thus, we sought to formulate and validate a nomogram prediction model for POPF after PD and the development of a novel strategy for intraoperative placement and early removal of prophylactic drains. We present the following article in accordance with the TRIPOD reporting checklist (available at https://gs.amegroups.com/article/ view/10.21037/gs-21-550/rc).

\section{Methods}

\section{Patients and methods}

A consecutive of 993 patients undergoing PD between January 2012 and December 2016 at the Pancreas Center of the First Affiliated Hospital with Nanjing Medical University were retrospectively in this study. All data were extracted and events were judged based on patients' medical records. Preoperative variables included patient demographics information, medical history, laboratory tests, and preoperative biliary drainage. Intraoperative variables included type of surgery (pylorus-preserving $v s$. classical), pancreatic duct diameter, combined organ resection, combined portal-superior mesenteric vein resection, duration of surgery, estimated blood loss, blood transfusion, intraoperative fluid infusion, and intraoperative diagnosis [pancreatic ductal adenocarcinoma (PDAC)/ chronic pancreatitis (CP) vs. others]. Postoperative variables included drainage fluid amylase (DFA) and absolute neutrophil count both on POD1 and POD3.

The entire cohort was randomly divided into training (662 patients) and validation (331 patients) group on a scale of 2:1 by $\mathrm{R}$ software. Intraoperative and postoperative nomogram models of POPF were respectively formulated based on several independent predictors which were obtained by univariable and multivariable analysis in the training cohort. The performance of models was estimated by internal validation in the training cohort and simultaneously verified by external validation in the validation cohort. According to the nomogram models, a risk-stratified drain management protocol with the purpose of drain omission (based on model I) and early drain removal (model II) for each patient was proposed.

\section{Definitions}

Pancreatic fistula was defined according to 2016 updated ISGPS criteria $(17,18)$. Biochemical fistula was characterized by elevated DFA levels ( $>3$ times the upper limit of the normal serum level), and required little to no deviation from the normal clinical pathway. Grade B fistula was typically treated with antibiotics, prophylactic 
somatostatin analogues, total parenteral nutrition, and percutaneous drain placement. Grade C fistula was characterized by organ failure and frequently required more aggressive treatment, including reoperation and intensive care unit (ICU) stay.

Intraoperative fluid administration included all the crystal and colloid fluids administered intravenously during the period from induction of general anesthesia to returning to ward of the patient. Blood product (packed red blood cells, fresh frozen plasma, platelet units or albumin) was not included in the calculation of the intraoperative colloid fluid volumes. Diameter of the pancreatic duct was measured intraoperatively after pancreas transection, rather than from the measurements in preoperative CT scan due to lack of data from the imaging study.

\section{Surgical procedure and drain management}

Classic Whipple or pylorus-preserving PD was performed according to the indication as well as preference of the individual surgeon. An internal stent was routinely placed in cases with normal diameter. No external stent was used in all cases. Pancreaticojejunostomy (PJ) was performed using two different methods: two-layer invagination and modified one-layer duct-to-mucosa which was reported in our previous study (19). During PJ, either interrupted or running suturing was adopted, depending on the surgeon's preference. Following PJ anastomosis, end-to-side single layer hepaticojejunostomy without stenting was performed in a continuous manner using the same jejunal loop (child's reconstruction). An antecolic gastrojejunostomy or duodenojejunostomy was constructed by hand or using a circular stapler.

Octreotide was routinely used until POD5 in all cases. During the study period, intraoperative drainage tubes were routinely placed in situ. DFA levels were routinely measured on or after POD3. Due to pressure erosions with intraluminal migration of a drain catheter may occur at the site of a pre-existing anastomotic leak, drains were pulled out 3 to $4 \mathrm{~cm}$ when DFA in POD3 -5 was normal $(\leq 330 \mathrm{U} / \mathrm{L})$, and drains were left in in place until either DFA was normal and/or the output was $10 \mathrm{~mL} /$ day or lesser for 3 consecutive days. The study was conducted in accordance with the Declaration of Helsinki (as revised in 2013). The study was approved by the First Affiliated Hospital with Nanjing Medical University Ethics Committee (No. 2018-SR-070) and individual consent for this retrospective analysis was waived.

\section{Statistical analysis}

Categorical variables were presented as frequency (percentage) and compared using a chi-square or Fisher's exact test. On the basis of whether the data was normally distributed, continuous data was described as the mean and standard deviation (SD) or median and range, and compared using a Student's $t$-test or Mann-Whitney U test accordingly. To identify intraoperative predictors of POPF, a univariable and multivariable logistic regression was performed. This procedure was repeated for postoperative predictors of POPF. The results are shown as odds ratio (OR) and 95\% confidence interval (CI). To determine predictors for POPF, a stepwise, backwards selection with a $\mathrm{P}$ value of $<0.05$ was used to select each variable from the multivariable logistic regression analysis. From the ORs of the significant predictors of the multivariable logistic regression, a nomogram was established. The highest $\beta$ coefficient of each variable was proportionally converting to a 0 to 100 points scale. The points of each variable were then added up to derive a total 200 points scale, which were finally converted to predict individual probabilities of POPF. Model performance was assessed by measurements of discrimination and calibration. Discrimination measures the ability to separate patients who experience POPF from those who will not. Calibration measures the ability to correctly quantify the observed absolute risk. All missing data were excluded in our nomograms and validation of models. A two tailed $\mathrm{P}<0.05$ was considered statistically significant. All statistical analyses were carried out using STATA 10.0 for Windows (StataCorp LP, College Station, TX, USA). The nomogram was established using $\mathrm{R}$ software version 3.3.2 (R foundation for Statistical Computing, Vienna, Austria; http://www.R-project.org/).

\section{Results}

\section{Overview}

Data from 993 patients (620 males and 373 females) who underwent PD during the study period were retrospectively analyzed. A total of 348 patients (35.0\%) had a history of hypertension and 138 patients $(13.9 \%)$ had diabetes mellitus. Preoperative biliary drainage was performed in 103 patients (10.4\%). A total of 590 patients (59.4\%) underwent classical PD, and the remaining 403 patients (40.6\%) underwent pylorus preserving pancreaticoduodenectomy (PPPD). Portal-superior mesenteric vein resection was performed in 72 patients $(7.3 \%)$ and combined organ 
resection was performed in 114 patients (11.5\%). The median operative blood loss was $300 \mathrm{~mL}$ (range, 50-2,800 mL) and median surgical duration was 245 minutes (range, 90-660 minutes). Two hundred and ninety-five patients (29.7\%) received red blood cell transfusion, and 283 patients $(28.5 \%)$ received plasma transfusion. The median intraoperative crystal and colloid infusion was $1,500 \mathrm{~mL}$ and 1,000 $\mathrm{mL}$ (range, 500-4,000 $\mathrm{mL}$ and 0-2,500 mL).

Postoperatively, the median DFA on postoperative day 3 was $113 \mathrm{U} / \mathrm{L}$ (range, 6-38,912 U/L) and median postoperative day 3 blood neutrophil count was $7.3 \times 10^{9} / \mathrm{L}$ [range, $(1.33-33.5) \times 10^{9} / \mathrm{L}$ ]. A total of 162 patients $(16.3 \%)$ developed POPF (grade B $+\mathrm{C}$ ). Of those with POPF, 146 patients $(14.7 \%)$ had grade B POPF, and 16 (1.6\%) patients had grade $\mathrm{C}$ POPF. The median hospital stay was 18 days (range, 5-132 days). Fourteen patients (1.4\%) demised in hospital due to severe complications after surgery.

Patients were randomly assigned to one of two cohorts: a training cohort $(n=662)$ and a validation cohort $(n=331)$. The baseline characteristics of the cohorts were summarized in Table 1.

\section{Predictors of POPF}

Logistic regression analysis was performed to identify risk factors of POPF in the cohort of all patients. Table 1 showed the results of the univariable analysis of 23 parameters examined as potential risk factors for the 162 patients with POPF compared with the 831 patients classified as nonPOPF. Lower preoperative alanine transaminase (ALT) level, smaller pancreatic duct diameter, no need for portalsuperior mesenteric vein resection, and pathology other than PDAC or CP were associated with the development of POPF. Postoperatively, higher DFA and higher neutrophil count on POD3 were found to be risk factors with statistical significance.

\section{Model I (intraoperative)}

Univariable analysis was used to determine the predictive value of pre-and intra-operative risk factors for POPF in the training cohort $(n=662)$. In the multivariable logistic regression analysis, preoperative ALT (OR 1.00, 95\% CI: 1.00-1.00, $\mathrm{P}=0.023$ ), need for vein resection (OR 0.22, 95\% CI: $0.05-0.95, \mathrm{P}=0.043)$, pancreatic duct diameter (OR 1.48, 95\% CI: 1.11-1.96, $\mathrm{P}=0.007$ ), intraoperative colloid infusion (OR 1.00, 95\% CI: 1.00-1.00, P=0.001), and pathology (OR 1.71, 95\% CI: 1.09-2.66, $\mathrm{P}=0.018)$ were factors independently associated with POPF (Table 2). A nomogram model $(n=655$, excluding 7 cases with missing data) was established based on independent pre-/intraoperative predictors identified in the multivariable analysis. Patient score points were calculated for the presence or absence of these factors. Total points can be translated into the probability of developing POPF after PD (Figure 1).

Internal validation was performed, and area under the receiver operating characteristic (ROC) curve (AUC) of POPF prediction with model I was 0.667. The AUC of FRS in external validation cohort was 0.683 (Figure 2). The calibration plot showed good correlation between the expected risk and the patient's actual risk in the low-risk range (risk probability range, $0.05-0.15$ ). In the external validation ( $n=327$, excluding 4 cases with missing data), AUC of the ROC curve was 0.621 and the calibration curve also showed good performance in the low-risk range (Figure 3).

\section{Model II (postoperative)}

Univariable analysis were performed for predictors of POPF on POD3. Pancreatic duct diameter (OR 1.58, 95\% CI: 1.14-2.19, $\mathrm{P}=0.006)$, intraoperative colloid infusion (OR 2.52, 95\% CI: 1.26-5.06, P=0.009), POD3 DFA (OR 4.70, 95\% CI: 3.30-6.70, $\mathrm{P}<0.001)$, and POD3 blood neutrophil count (OR 2.83, 95\% CI: 1.63-4.93, P<0.001) were significant factors for POPF prediction in the multivariable analysis (Table 3). Nomogram predicting for POPF was constructed using these 4 variables with different weights (Figure 4).

Internal validation $(n=440$, excluding 221 cases with missing data) and external validation $(n=205$, excluding 126 cases with missing data) were carried out. AUC for the training cohort was 0.809 and 0.797 for the validation cohort. The calibration plots in both internal and external validation showed good correlation between the expected risk and patient's actual risk in the low-risk range (Figure 5).

\section{Risk-stratified strategy for drain management}

According to the nomogram for prediction of POPF, we proposed a risk-stratified drain management protocol with the purpose of drainage omission (based on model I) and early drain removal (model II) for each patient (Figure 6). We set the predicted POPF risk probability $=0.05$ and 0.1 as cut-off values in two models, respectively. With missing data excluded, 646 patients in the full cohort were enrolled in the strategy performance evaluation. Intraoperatively, 
Table 1 Comparison of baseline data between the training cohort and the validation cohort, and between the POPF and non-POPF group

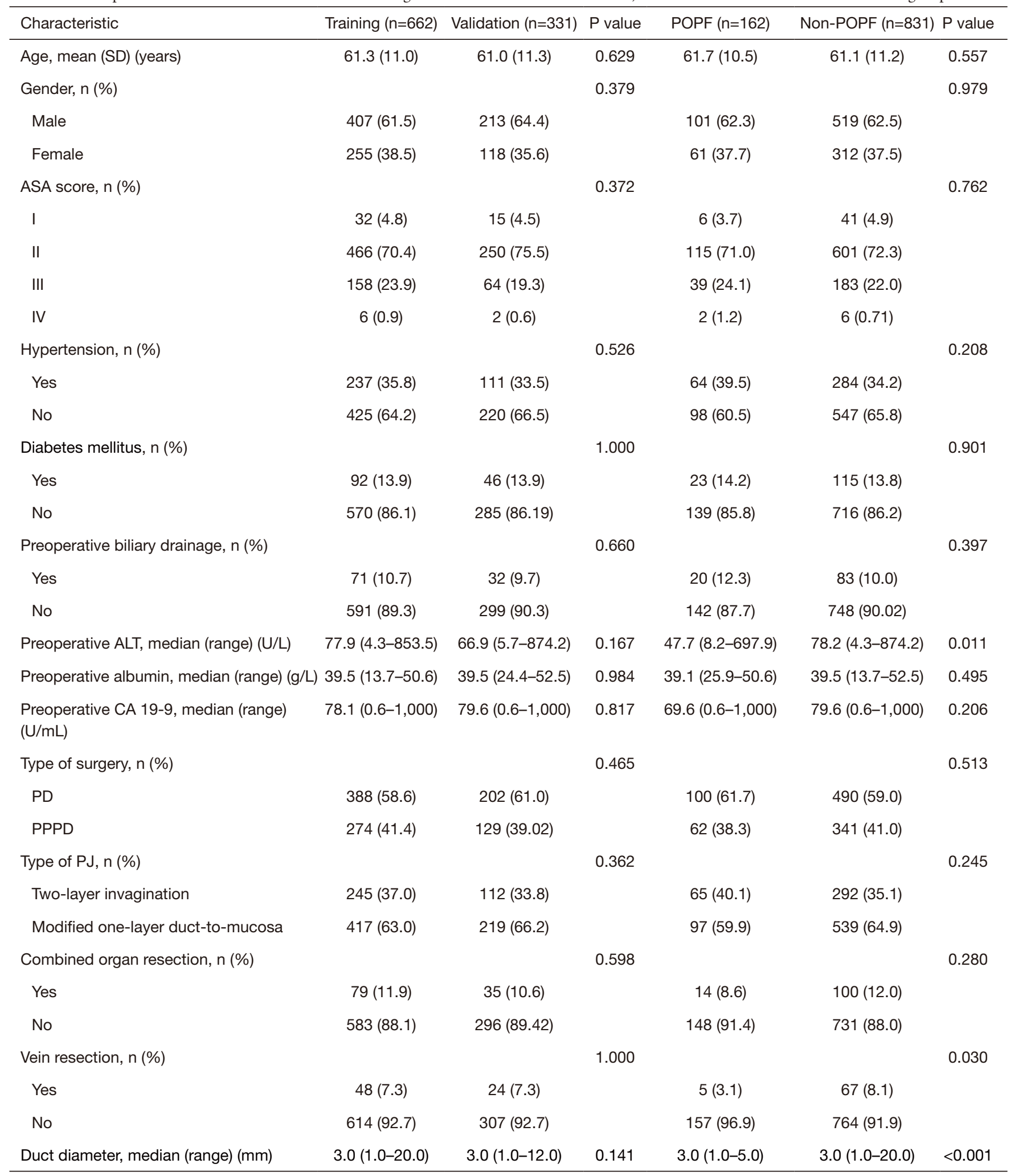

Table 1 (continued) 
Table 1 (continued)

\begin{tabular}{|c|c|c|c|c|c|c|}
\hline Characteristic & Training $(n=662)$ & Validation $(n=331)$ & $P$ value & POPF $(n=162)$ & Non-POPF $(n=831)$ & $P$ value \\
\hline$<2$ & $11(1.7)$ & $4(1.2)$ & & $4(2.5)$ & $11(1.3)$ & \\
\hline$\geq 2,<3$ & $178(26.9)$ & $113(34.1)$ & & $73(45.1)$ & $220(26.5)$ & \\
\hline$\geq 3,<4$ & 317 (47.9) & $147(44.4)$ & & $62(38.3)$ & $404(48.6)$ & \\
\hline Duration of surgery, median (range) (min) & $250(90-545)$ & $240(105-660)$ & 0.498 & $245(105-531)$ & $245(90-660)$ & 0.859 \\
\hline Estimated blood loss, median (range) $(\mathrm{mL})$ & $300(50-2,300)$ & $300(50-2,800)$ & 0.532 & $300(50-1,500)$ & $300(50-2,800)$ & 0.655 \\
\hline $\begin{array}{l}\text { Intraoperative colloid infusion, median } \\
(\text { range) }(\mathrm{mL})\end{array}$ & $1,000(0-2,500)$ & $1,000(0-2,500)$ & 0.746 & $1,000(0-2,500)$ & $1,000(0-2,500)$ & 0.136 \\
\hline PDAC or CP & $331(50.0)$ & $167(50.5)$ & & $56(34.6)$ & $442(53.2)$ & \\
\hline Others & $331(50.0)$ & $164(49.5)$ & & $106(65.4)$ & $339(40.8)$ & \\
\hline POD3 DFA, median (range) $(\mathrm{U} / \mathrm{L})^{\star}$ & $105(6-38,912)$ & $163(25-30,000)$ & 0.167 & $1,200.5(30-38,912)$ & $78(6-30,000)$ & $<0.001$ \\
\hline POD3 DFA, median (range) (U/L) ${ }^{\star}, \mathrm{n}(\%)$ & & & 0.138 & & & $<0.001$ \\
\hline$<600$ & $336(68.2)$ & $177(75.0)$ & & $32(26.0)$ & $481(79.4)$ & \\
\hline $600-2,000$ & $121(24.5)$ & $43(18.2)$ & & $59(48.0)$ & $105(17.3)$ & \\
\hline$\geq 2,000$ & $36(7.3)$ & $16(6.81)$ & & $32(26.0)$ & $20(3.31)$ & \\
\hline
\end{tabular}

if the predicted POPF risk in model $\mathrm{I}<0.05$, prophylactic drainage would be omitted in 64 patients $(9.9 \%)$. Otherwise, prophylactic drainage would be used. The rest 582 patients $(90.1 \%)$ with intraoperatively placed drainage in our virtual strategy application were then enrolled in model II risk prediction which can guide early drain removal on POD3. Similarly, if the predicted risk $<0.1$ in model II, 171 patients $(26.5 \%)$ would be suitable for early drain removal. In our simulated examination, 4 patients without drainage and 8 patients with early drain removal actually developed POPF (12/646, $1.9 \%$ in total). Among the remaining 411 patients (63.6\%) with drains placed in situ, there were 102 patients (15.8\%) suffered POPF eventually. The sensitivity and specificity of POPF for this risk-stratified approach was 0.895 and 0.419 . The sensitivity and negative predict value (NPV) of the twostep prediction method were 0.898 and 0.949 , respectively. A total of 223 patients (34.5\%) whose drainage was omitted or removed early would benefit from our new risk-based drainage management strategy. 
Table 2 Univariable and multivariable analysis of pre- and intra-operative predictors of POPF in the training cohort

\begin{tabular}{|c|c|c|c|c|c|c|}
\hline Characteristic & \multicolumn{3}{|c|}{ Univariate } & \multicolumn{3}{|c|}{ Multivariate } \\
\hline Preoperative ALT & 0.998 & $0.667-0.999$ & 0.002 & 1.00 & $1.00-1.00$ & 0.023 \\
\hline Vein resection & 0.35 & $0.14-0.90$ & 0.029 & 0.22 & $0.05-0.95$ & 0.043 \\
\hline Duct diameter (mm) & & & & 1.48 & $1.11-1.96$ & 0.007 \\
\hline$\geq 2,<3$ & 0.93 & $0.29-2.99$ & 0.897 & & & \\
\hline$\geq 3,<4$ & 0.43 & $0.13-1.38$ & 0.154 & & & \\
\hline$\geq 4$ & 0.32 & $0.09-1.10$ & 0.070 & & & \\
\hline Intraoperative colloid infusion & 1.00 & $1.00-1.00$ & 0.039 & 1.00 & $1.00-1.00$ & 0.001 \\
\hline
\end{tabular}

OR, odds ratio; $\mathrm{Cl}$, confidence interval; ALT, alanine transaminase; PDAC, pancreatic ductal adenocarcinoma; CP, chronic pancreatitis; POPF, postoperative pancreatic fistula.

Points
Venous resection
Pathology
Duct diameter (mm)
Intraoperative colloid infusion (L)
Preoperative ALT (x $100 \mathrm{U} / \mathrm{L}$ )

Figure 1 Model I (pre-/intra-operative) nomogram for prediction of POPF after PD. Patient score points were calculated for the presence or absence of the factors. Total points were translated into the probability of developing POPF. POPF, postoperative pancreatic fistula; CP, chronic pancreatitis; PD, pancreaticoduodenectomy; ALT, alanine transaminase.

\section{Discussion}

POPF remains the major cause of morbidity and mortality after PD (3-5). The rate of POPF in our study was $16.3 \%$ and this is within the expected range when compared with the existing literature $(12,20-22)$. The ability to predict the development of POPF enables more individualized surgical management of patients after PD. Currently, six models have been reported for POPF risk prediction after $\mathrm{PD}$ procedures using 16 predictors, although most have not been well validated $(23,24)$. FRS has been established as a reliable intraoperative predictor of POPF based on the following risk factors: soft gland parenchyma, high risk pathology, small duct diameter, and elevated intraoperative blood loss. Individual FRS scores are discretized and assigned to one of four risk zones. The result of internal 


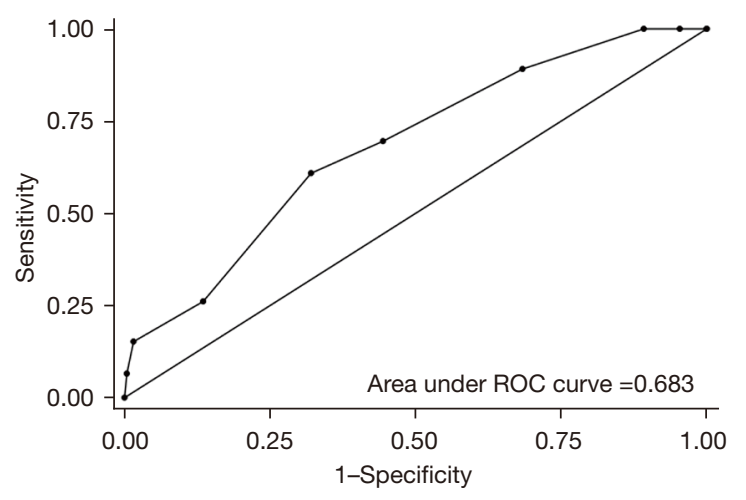

Figure 2 ROC curve of FRS in external validation cohort. The area under ROC curve was 0.683. FRS, Fistula Risk Score; ROC, receiver operating characteristic.

validation for FRS showed a relative high AUC that was 0.942 (11). Although, numerous studies regarding FRS or modified FRS confirmed the feasibility and validity for prediction of POPF $(13,25,26)$, when we used FRS to perform a validation in our validation cohort, the AUC was 0.683 which meant FRS was not an optimal model for our practice. A moderate/high risk patient with POD1 DFA $\leq 5,000 \mathrm{U} / \mathrm{L}$, a strong predictor for POPF, was nominated for early drain removal on POD3. Although, approximately $20 \%$ of patients with a POPF rate of $38.2 \%$ kept drain at surgeon's discretion (12). Compliance with this approach dogmatically was extremely aggressive and may be detrimental to the patients. In light of those findings, this study sought to develop a personal risk prediction model for POPF after PD and propose a novel risk-stratified strategy for intraoperative placement and/or early removal of prophylactic drainage.

In our present study, we first developed two nomograms based on the following predictive factors: preoperative ALT, portal-superior mesenteric vein resection, pancreatic duct diameter, intraoperative colloid infusion, pathology, POD3 DFA, and neutrophil count on POD3. Normal ALT levels have been reported as a risk factor of POPF in our previous study (27), and the current study confirm it as a robust predictor for POPF, though the study population of the previous report was fully included in current study. However, the underlining mechanism to explain this statistical association is largely unknown to us, as elevated ALT before surgery always related to baseline liver disease (e.g., cirrhosis) and biliary obstruction. Relatively normal liver function was mostly observed in patients with a normal pancreas and no history of jaundice, which has been reported as risk factors of POPF $(28,29)$. It is improper to simply conclude that preoperative improvement in liver function will increase POPF rate; on the contrary, conditions including cirrhosis and portal hypertension may compromise surgical outcomes (30). In a word, the role of ALT as a preoperative POPF predictor still warrant external validation in patient cohorts outside our institution. The need for vein resections was frequently seen in patients with pancreatic cancer with larger tumor, implying more severe chronic inflammation changes in the pancreas, which was a recognized strong protective factor of POPF (31). Precise fluid management considering measurements of all fluid losses and the loss-to-third space was proven to be associated with better outcomes including less POPF after PD $(32,33)$. Greater volume of infused colloid fluid often suggests more blood loss, unstable intraoperative hemodynamic status and longer duration of surgery, which were reported to be associated with increased risk of POPF. Besides, colloid fluid overload with consequent parenchymal edema and local inflammation may be the mechanistic causes of increased POPF following PD. Pancreatic texture and duct size were widely reported and accepted as strong predictive factors for POPF in previous studies (24). However, the evaluation of parenchymal consistency was subjective and its reliability in the retrospective study was even more questionable. So, only pancreatic duct, but not pancreatic texture was analyzed in our present study. Pathological fibrosis and acinar cell content at the pancreatic stump, which is closely associated with pancreatic consistency, were also reported to be predictive for POPF (34). A histological score method based on the frozen sections was reported to well predict the risk of POPF development (35). However, these factors were also excluded from this study subjecting to the difficulty in our institution.

For model I, AUC for the training cohort was 0.667 and that for the validation cohort was 0.621 . This result was similar to the recent study (16). In addition, calibration plot showed a good accordance between the expected risk and patient's actual risk in the range of 0.05 to 0.15 , thus providing a good predictive value in identifying patients with lowest risk of POPF. In other words, our nomogram model I would have a good performance when the predicted POPF probability less than a certain cut-off value at low range was used to select patients for drainage omission. Similar characteristic was found in model II.

Routine drain placement after PD is a textbook practice during pancreatic surgery. However, the risks and benefits of routine drainage remain a source of controversy in the 

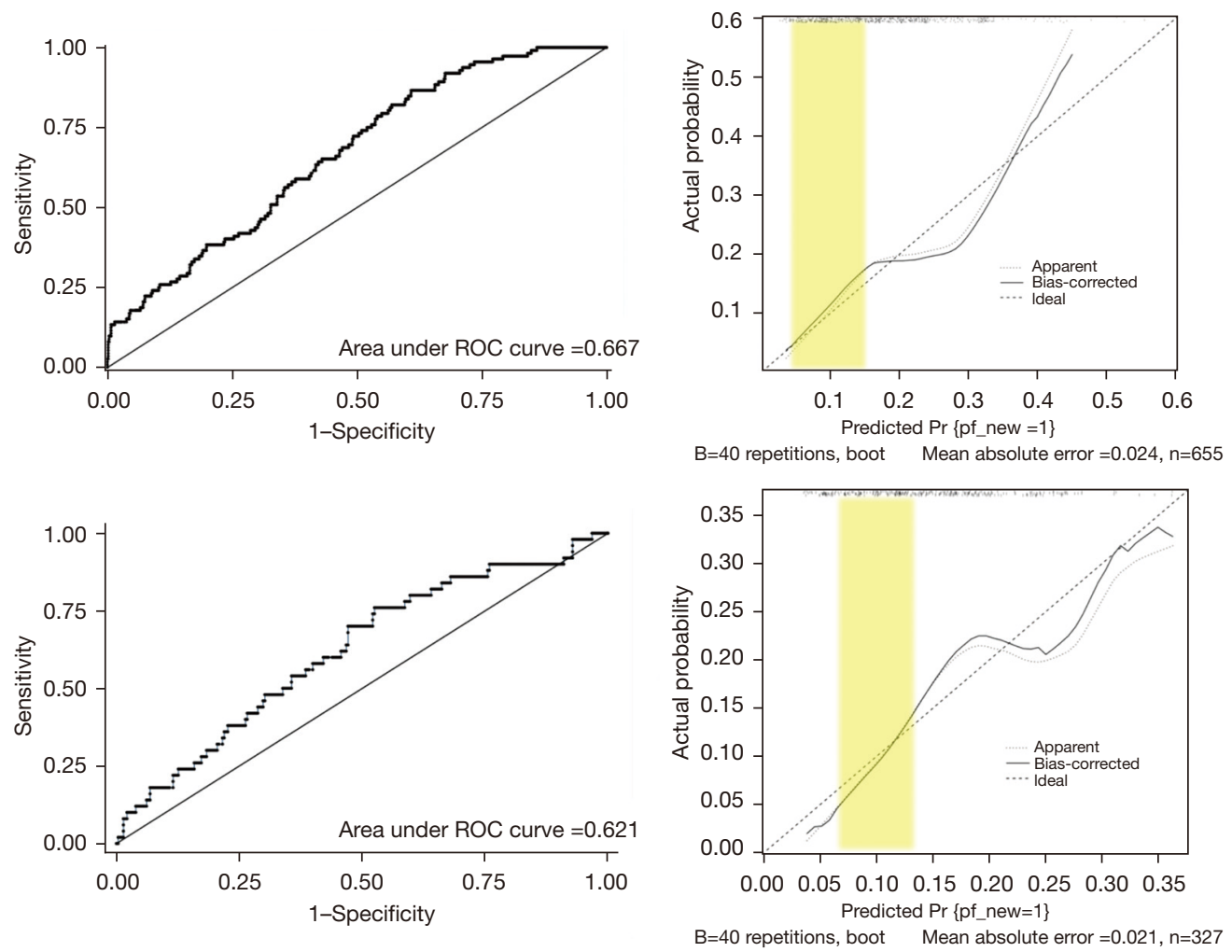

Figure 3 Internal and external validation of model I (pre-/intra-operative). ROC curve and calibration plot for nomogram. The area under ROC curve of POPF prediction with model I was 0.667 for internal validation, and 0.621 for external validation. The calibration plots showed good correlation between the expected risk and the patient's actual risk in the low-risk range (yellow band). POPF, postoperative pancreatic fistula; ROC, receiver operating characteristic.

Table 3 Univariable and multivariable analysis of postoperative predictors of POPF in the training cohort

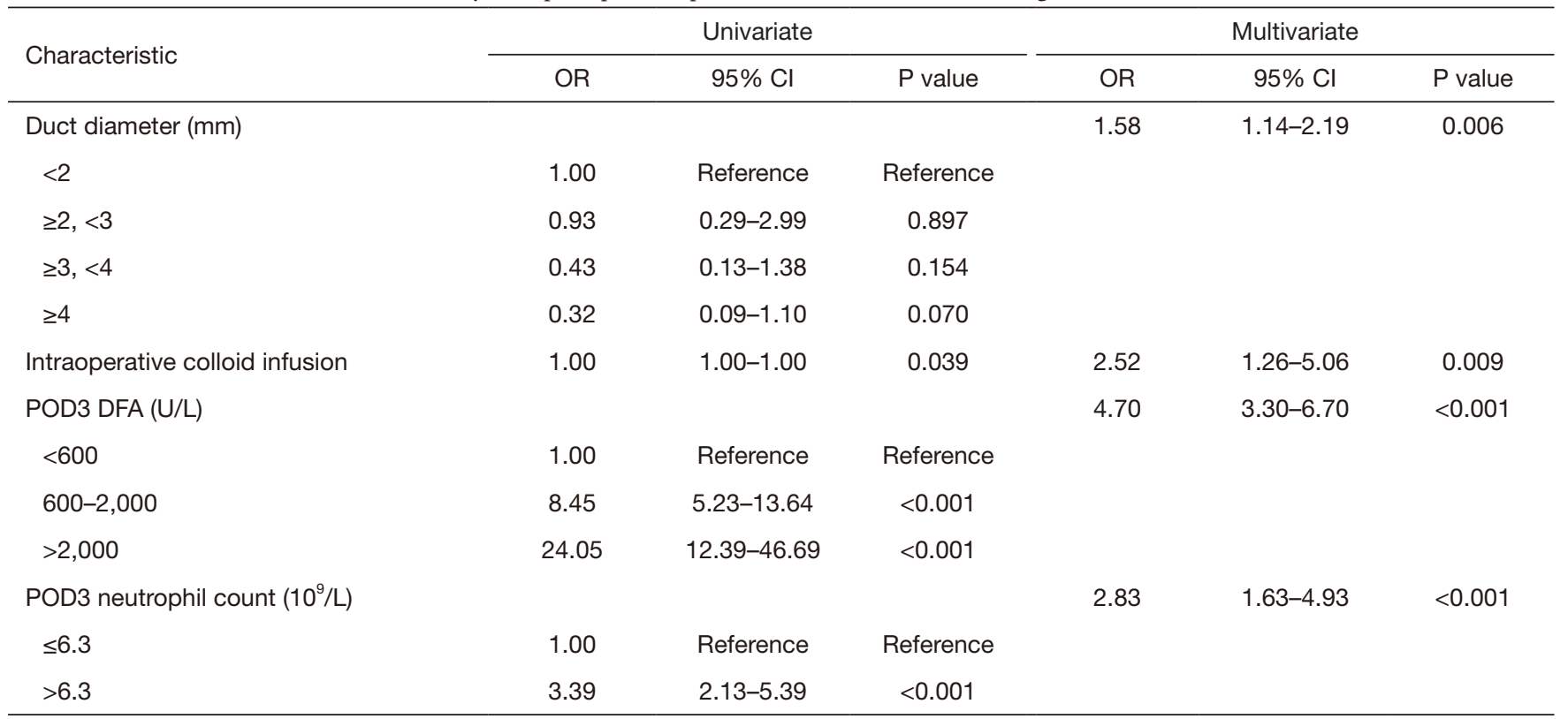

POPF, postoperative pancreatic fistula; OR, odds ratio; $\mathrm{Cl}$, confidence interval; POD3, postoperative day 3; DFA, drainage fluid amylase. 
Points

Duct diameter (mm)

Intraoperative colloid infusion (L)

POD3 DFA (U/L)

POD3 neutrophil count $\left(10^{9} / \mathrm{L}\right)$

Total points

Risk

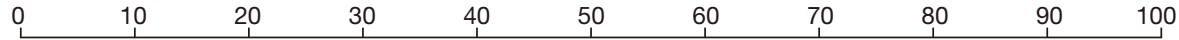

$\begin{array}{rrrrr} & 0.8 & 1.5 & & 2.5 \\ 0.5 & & 1 & & 2\end{array}$

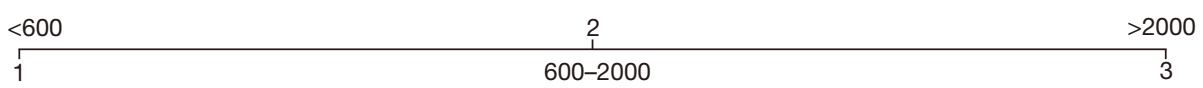

\section{5}
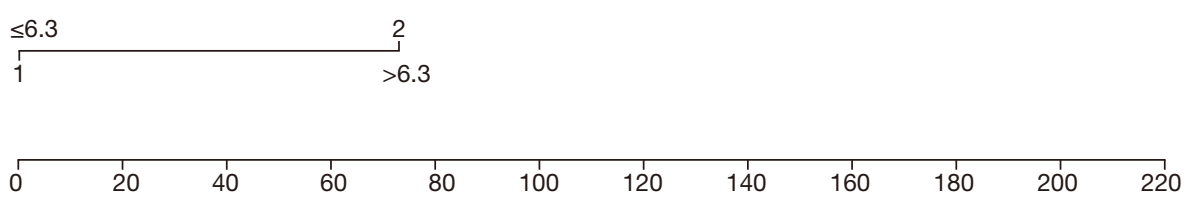

$\begin{array}{lllllllll}0.1 & 0.2 & 0.3 & 0.4 & 0.5 & 0.6 & 0.7 & 0.8 & 0.9\end{array}$

Figure 4 Model II (postoperative) nomogram for prediction of POPF after PD. POPF, postoperative pancreatic fistula; PD, pancreaticoduodenectomy; DFA, drain fluid amylase.
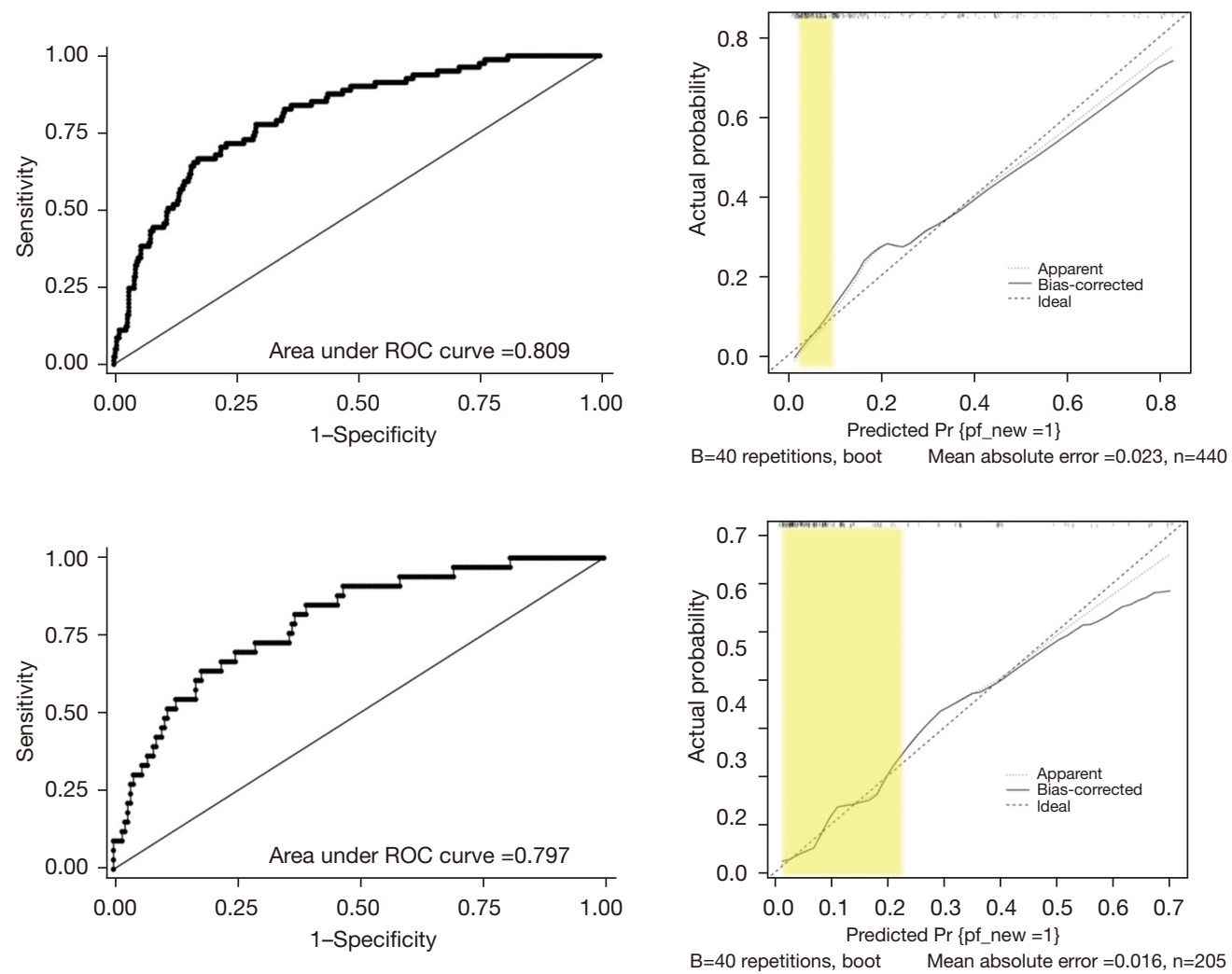

Figure 5 Internal and external validation of model II (postoperative). ROC curve and calibration plot for nomogram. The area under ROC curve of POPF prediction with model II was 0.809 for internal validation, and 0.797 for external validation. The calibration plots showed good correlation between the expected risk and the patient's actual risk in the low-risk range (yellow band). POPF, postoperative pancreatic fistula; ROC, receiver operating characteristic. 


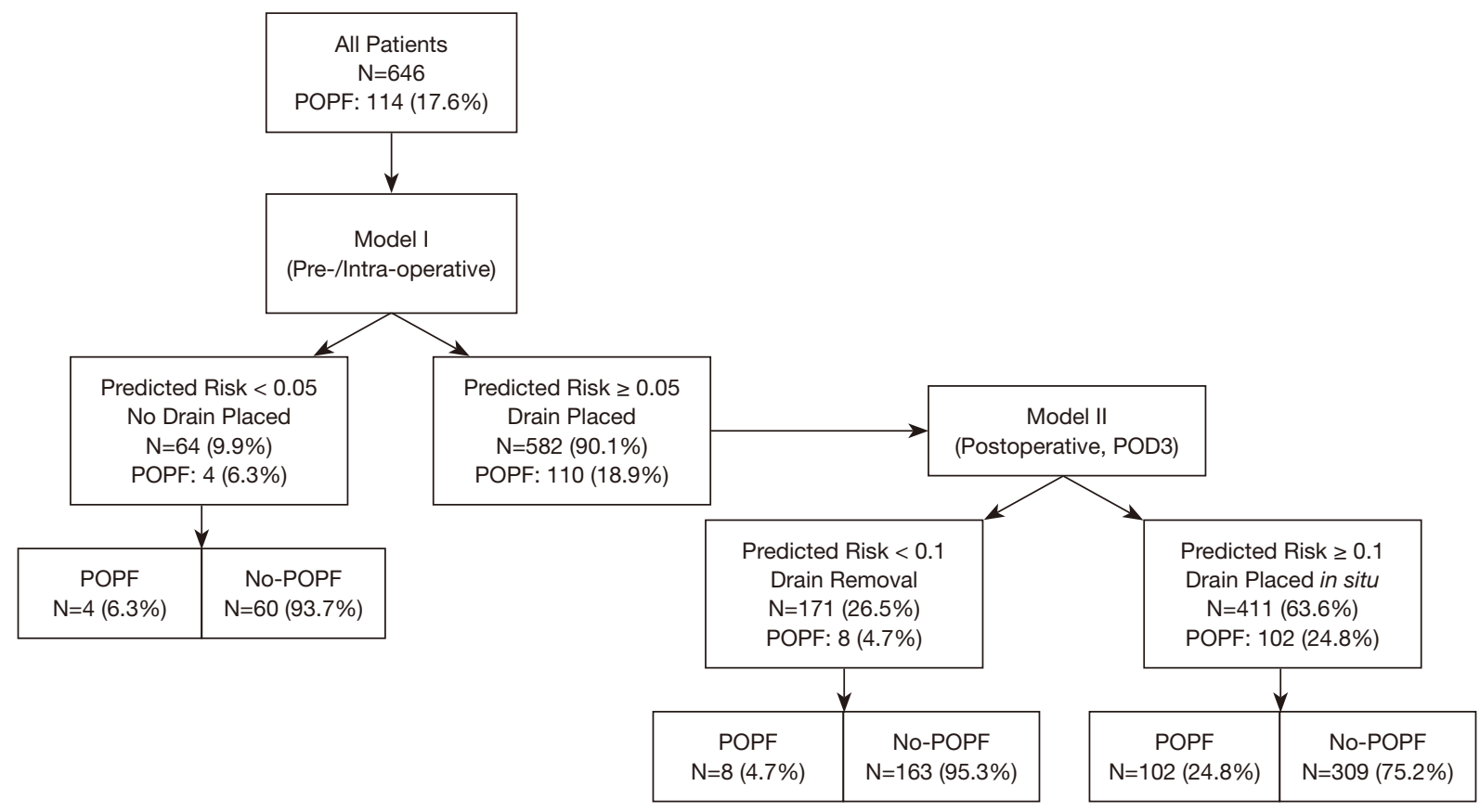

Figure 6 A risk-stratified drain management strategy with the purpose of drainage omission and early drain removal based on the two nomogram models for prediction of POPF. With missing data excluded, 646 patients in the full cohort were enrolled in the strategy performance simulated evaluation. If the predicted risk was $<0.05$, no drain would be placed in model I. Otherwise, drain would be placed and moved to model II. If the predicted risk was $<0.1$ on POD3, the drain would be removed early. Through the comparison of the predicted and actual POPF occurrence pattern, a total of 223 patients (34.5\%) would theoretically benefit from our strategy. POPF, postoperative pancreatic fistula; POD, postoperative day.

literature (1,8,36-40). Proponents of this practice cite the ability to evacuate bile, chyle, blood, and pancreatic juice that may accumulate during the postoperative period. Together with the option of controlled drainage, in the case of an existing fistula, there is a reliance on abdominal drainage after pancreatic surgery to detect and minimize postoperative hemorrhage arising from the erosion of retroperitoneal vessels (36). In a previous multicenter trial, decreased mortality and morbidity were associated with routine drainage after $\mathrm{PD}$, with a 4-fold increase in mortality from $3 \%$ to $12 \%$ following elimination of intraperitoneal drainage after pancreatectomy (1). However, opponents of routine drain believe that drains may provide access for bacteria, potentially leading to abdominal infections, and are frequently ineffective in controlling pancreatic leak or abscess and postoperative percutaneous drainage is still required (39-41). In a recent dual-center, randomized, controlled, non-inferiority trial among 395 patients (202 drain, 193 no-drain), the omission of drains was found to be not inferior to intraabdominal drainage in terms of postoperative re-intervention and was superior in terms of clinically relevant (CR) pancreatic fistula rate and fistula associated complications (8). Nonetheless, this result has been criticized because only $13 \%$ of eligible patients and 40 patients in the no-drain group had drains placed because of surgeons deliberately violating trial protocol, resulting in screening bias and selection bias. Generally, the question of routinely drain or selectively drain was subjected to actual situation of each institution regarding the experience of surgeons and radiologists, and that needed institutional dependent drain management. Therefore, drain management protocol based on POPF predictive model seemed more precise. In a recent multicenter prospective trial, the concept of selective drainage was practiced in patients with a negligible/low FRS. Moreover, moderate/high-risk patients with POD1 drain amylase $<5,000 \mathrm{U} / \mathrm{L}$ had lower rates of clinical relevant POPF with POD3 (vs. POD >5) drain removal $(7,12)$. The views about the management and timing of drain removal were gradually converged in up-to-date studies. It may be appropriate that operatively placed drains should be removed by POD3, because early drain removal when POD1 DFA was 
$\leq 5,000 \mathrm{U} / \mathrm{L}$ was associated with significant reductions in overall morbidity (35.3\% vs. $52.3 \%)$, CR-POPF (0.9\% vs. $7.9 \%$ ) (42). Given that, a recent systemic review proposed routine placement of a drain and early removal was the most conservative approach (43). In our clinical practice, we certainly believe that a portion of low-risk patients do not need drains.

Based on the predictive accuracy of POPF in low-risk populations, model I was used to determine to place drain or not. Then, combined POD3 DFA and neutrophil count, model II expectedly played a key role in early drain removal. That was the core of our risk stratified strategy for drain management. Taking full consideration of patient safety in our risk stratified approach, intraabdominal drainage can be omitted with $5 \%$ or lower rate of developing POPF, and the drain can be removed on POD3 with a $10 \%$ or lower rate of POPF occurrence. In model I, among near 10\% patients who assumed low risk of fistula, only 4 patients (6.3\%) actually suffered grade B POPF, which meant it was relative safe for drain omission in $9.3 \%$ patients. A repeated calculation combined POD3 DFA and neutrophil count for the remaining patients. Among them, 171 patients $(29.4 \%)$ were applicable to early drain removal, and 8 patients (4.7\%) developed POPF eventually. Accordingly, early drain removal on POD3 was acceptable in relative low risk patients. Through our nomograms, one third of patients would not need prophylactic drainages or could remove drains on POD3. Moreover, this protocol has an advantage in terms of balancing patient safety and benefit by proper alternation of low-risk cutoff value according to variability between institutions. This protocol can guide patient management, but it should not supplant surgeon judgment, especially in moderate and high-risk patients. The fact that we scarcely omitted drains after pancreatectomies or removed drains on POD3 in our practice. We hoped that this study could change our deep understanding of drains and overcome our overreliance on drains.

There were several drawbacks in this study. First, this study was carried out in a retrospective manner. It was difficult to exclude all information bias and confounding bias due to the nature of this study design. Second, the validity of our prediction models did not show a higher AUC as compared with FRS and lacked real external validation. Third, with hindsight, our risk-stratified strategy was idealistic and theoretically beneficial for one third of patients, however, that were not prospectively validated in our clinical practice.

In conclusion, we formulated and validated a nomogram model for predicting POPF that showed good performance, especially in the low-risk range. This tool may help surgeons to identify a risk stratified strategy for intraoperative drain placement and early drain removal. This unique approach may be beneficial for a substantial proportion of patients.

\section{Acknowledgments}

Funding: This study was funded by the National Natural Science Foundation of China (grant No. $81272382 \& 81672449)$, the Innovation Capability Development Project of Jiangsu Province (No. BM2015004 to $\mathrm{YM}$ ), and the Jiangsu Province Funding Program for Six Major Talent Summit, China (grant No. 2014-WSW-006).

\section{Footnote}

Reporting Checklist: The authors have completed the TRIPOD reporting checklist. Available at https:// gs.amegroups.com/article/view/10.21037/gs-21-550/rc

Data Sharing Statement: Available at https://gs.amegroups. com/article/view/10.21037/gs-21-550/dss

Peer Review File: Available at https://gs.amegroups.com/ article/view/10.21037/gs-21-550/prf

Conflicts of Interest: All authors have completed the ICMJE uniform disclosure form (available at https://gs.amegroups. com/article/view/10.21037/gs-21-550/coif). The authors have no conflicts of interest to declare.

Etbical Statement: The authors are accountable for all aspects of the work in ensuring that questions related to the accuracy or integrity of any part of the work are appropriately investigated and resolved. The study was conducted in accordance with the Declaration of Helsinki (as revised in 2013). The study was approved by the First Affiliated Hospital with Nanjing Medical University Ethics Committee (No. 2018-SR-070) and individual consent for this retrospective analysis was waived.

Open Access Statement: This is an Open Access article distributed in accordance with the Creative Commons Attribution-NonCommercial-NoDerivs 4.0 International License (CC BY-NC-ND 4.0), which permits the noncommercial replication and distribution of the article with the strict proviso that no changes or edits are made and the 
original work is properly cited (including links to both the formal publication through the relevant DOI and the license). See: https://creativecommons.org/licenses/by-nc-nd/4.0/.

\section{References}

1. Van Buren G 2nd, Bloomston M, Hughes SJ, et al. A randomized prospective multicenter trial of pancreaticoduodenectomy with and without routine intraperitoneal drainage. Ann Surg 2014;259:605-12.

2. Greenblatt DY, Kelly KJ, Rajamanickam V, et al. Preoperative factors predict perioperative morbidity and mortality after pancreaticoduodenectomy. Ann Surg Oncol 2011;18:2126-35.

3. Gouma DJ, van Geenen RC, van Gulik TM, et al. Rates of complications and death after pancreaticoduodenectomy: risk factors and the impact of hospital volume. Ann Surg 2000;232:786-95.

4. Pratt WB, Maithel SK, Vanounou T, et al. Clinical and economic validation of the International Study Group of Pancreatic Fistula (ISGPF) classification scheme. Ann Surg 2007;245:443-51.

5. van Berge Henegouwen MI, De Wit LT, Van Gulik TM, et al. Incidence, risk factors, and treatment of pancreatic leakage after pancreaticoduodenectomy: drainage versus resection of the pancreatic remnant. J Am Coll Surg 1997;185:18-24.

6. Vollmer CM Jr, Sanchez N, Gondek S, et al. A root-cause analysis of mortality following major pancreatectomy. $\mathrm{J}$ Gastrointest Surg 2012;16:89-102; discussion 102-3.

7. McMillan MT, Malleo G, Bassi C, et al. Multicenter, Prospective Trial of Selective Drain Management for Pancreatoduodenectomy Using Risk Stratification. Ann Surg 2017;265:1209-18.

8. Witzigmann H, Diener MK, Kienkötter S, et al. No Need for Routine Drainage After Pancreatic Head Resection: The Dual-Center, Randomized, Controlled PANDRA Trial (ISRCTN04937707). Ann Surg 2016;264:528-37.

9. Kawai M, Tani M, Terasawa H, et al. Early removal of prophylactic drains reduces the risk of intra-abdominal infections in patients with pancreatic head resection: prospective study for 104 consecutive patients. Ann Surg 2006;244:1-7.

10. Ven Fong Z, Correa-Gallego C, Ferrone CR, et al. Early Drain Removal--The Middle Ground Between the Drain Versus No Drain Debate in Patients Undergoing Pancreaticoduodenectomy: A Prospective Validation Study. Ann Surg 2015;262:378-83.
11. Callery MP, Pratt WB, Kent TS, et al. A prospectively validated clinical risk score accurately predicts pancreatic fistula after pancreatoduodenectomy. J Am Coll Surg 2013;216:1-14.

12. McMillan MT, Malleo G, Bassi C, et al. Drain Management after Pancreatoduodenectomy: Reappraisal of a Prospective Randomized Trial Using Risk Stratification. J Am Coll Surg 2015;221:798-809.

13. Mungroop TH, van Rijssen LB, van Klaveren D, et al. Alternative Fistula Risk Score for Pancreatoduodenectomy (a-FRS): Design and International External Validation. Ann Surg 2019;269:937-43.

14. Hogg ME, Zenati M, Novak S, et al. Grading of Surgeon Technical Performance Predicts Postoperative Pancreatic Fistula for Pancreaticoduodenectomy Independent of Patient-related Variables. Ann Surg 2016;264:482-91.

15. Moons KG, Royston P, Vergouwe Y, et al. Prognosis and prognostic research: what, why, and how? BMJ 2009;338:b375.

16. You Y, Han IW, Choi DW, et al. Nomogram for predicting postoperative pancreatic fistula. HPB (Oxford) 2019;21:1436-45.

17. Bassi C, Dervenis C, Butturini G, et al. Postoperative pancreatic fistula: an international study group (ISGPF) definition. Surgery 2005;138:8-13.

18. Bassi C, Marchegiani G, Dervenis C, et al. The 2016 update of the International Study Group (ISGPS) definition and grading of postoperative pancreatic fistula: 11 Years After. Surgery 2017;161:584-91.

19. Wei J, Liu X, Wu J, et al. Modified One-layer Ductto-mucosa Pancreaticojejunostomy Reduces Pancreatic Fistula After Pancreaticoduodenectomy. Int Surg 2015. [Epub ahead of print].

20. Miller BC, Christein JD, Behrman SW, et al. A multiinstitutional external validation of the fistula risk score for pancreatoduodenectomy. J Gastrointest Surg 2014;18:17279; discussion 179-80.

21. Molinari E, Bassi C, Salvia R, et al. Amylase value in drains after pancreatic resection as predictive factor of postoperative pancreatic fistula: results of a prospective study in 137 patients. Ann Surg 2007;246:281-7.

22. Kimura W, Miyata H, Gotoh M, et al. A pancreaticoduodenectomy risk model derived from 8575 cases from a national single-race population (Japanese) using a web-based data entry system: the 30-day and inhospital mortality rates for pancreaticoduodenectomy. Ann Surg 2014;259:773-80.

23. Ramacciato G, Mercantini P, Petrucciani N, et al. Risk 
factors of pancreatic fistula after pancreaticoduodenectomy: a collective review. Am Surg 2011;77:257-69.

24. Vallance AE, Young AL, Macutkiewicz C, et al. Calculating the risk of a pancreatic fistula after a pancreaticoduodenectomy: a systematic review. HPB (Oxford) 2015;17:1040-8.

25. Ryu Y, Shin SH, Park DJ, et al. Validation of original and alternative fistula risk scores in postoperative pancreatic fistula. J Hepatobiliary Pancreat Sci 2019;26:354-9.

26. Kantor O, Talamonti MS, Pitt HA, et al. Using the NSQIP Pancreatic Demonstration Project to Derive a Modified Fistula Risk Score for Preoperative Risk Stratification in Patients Undergoing Pancreaticoduodenectomy. J Am Coll Surg 2017;224:816-25.

27. Yin J, Lu Z, Wu P, et al. Afferent Loop Decompression Technique is Associated with a Reduction in Pancreatic Fistula Following Pancreaticoduodenectomy. World J Surg 2018;42:3726-35.

28. Yu L, Huang Q, Xie F, et al. Risk factors of postoperative complications of pancreatoduodenectomy. Hepatogastroenterology 2014;61:2091-5.

29. Ausania F, Snowden CP, Prentis JM, et al. Effects of low cardiopulmonary reserve on pancreatic leak following pancreaticoduodenectomy. Br J Surg 2012;99:1290-4.

30. El Nakeeb A, Sultan AM, Salah T, et al. Impact of cirrhosis on surgical outcome after pancreaticoduodenectomy. World J Gastroenterol 2013;19:7129-37.

31. Sugimoto M, Takahashi S, Gotohda N, et al. Schematic pancreatic configuration: a risk assessment for postoperative pancreatic fistula after pancreaticoduodenectomy. J Gastrointest Surg 2013;17:1744-51.

32. Sandini M, Fernández-Del Castillo C, Ferrone CR, et al. Intraoperative Fluid Administration and Surgical Outcomes Following Pancreaticoduodenectomy: External Validation at a Tertiary Referral Center. World J Surg 2019;43:929-36.

33. Bruns H, Kortendieck V, Raab HR, et al. Intraoperative Fluid Excess Is a Risk Factor for Pancreatic Fistula after Partial Pancreaticoduodenectomy. HPB Surg

Cite this article as: Yin J, Zhu Q, Zhang K, Gao W, Wu J, Lu Z, Jiang K, Miao Y. Development and validation of risk prediction nomogram for pancreatic fistula and risk-stratified strategy for drainage management after pancreaticoduodenectomy. Gland Surg 2022;11(1):42-55. doi: 10.21037/gs-21-550
2016;2016:1601340.

34. Kiyochi H, Matsukage S, Nakamura T, et al. Pathologic Assessment of Pancreatic Fibrosis for Objective Prediction of Pancreatic Fistula and Management of Prophylactic Drain Removal After Pancreaticoduodenectomy. World J Surg 2015;39:2967-74.

35. Belyaev O, Munding J, Herzog T, et al. Histomorphological features of the pancreatic remnant as independent risk factors for postoperative pancreatic fistula: a matched-pairs analysis. Pancreatology 2011;11:516-24.

36. McMillan MT, Fisher WE, Van Buren G 2nd, et al. The value of drains as a fistula mitigation strategy for pancreatoduodenectomy: something for everyone? Results of a randomized prospective multi-institutional study. J Gastrointest Surg 2015;19:21-30; discussion 30-1.

37. Conlon KC, Labow D, Leung D, et al. Prospective randomized clinical trial of the value of intraperitoneal drainage after pancreatic resection. Ann Surg 2001;234:487-93; discussion 493-4.

38. van der Wilt AA, Coolsen MM, de Hingh IH, et al. To drain or not to drain: a cumulative meta-analysis of the use of routine abdominal drains after pancreatic resection. HPB (Oxford) 2013;15:337-44.

39. Correa-Gallego C, Brennan MF, D'angelica M, et al. Operative drainage following pancreatic resection: analysis of 1122 patients resected over 5 years at a single institution. Ann Surg 2013;258:1051-8.

40. Fisher WE, Hodges SE, Silberfein EJ, et al. Pancreatic resection without routine intraperitoneal drainage. HPB (Oxford) 2011;13:503-10.

41. Bassi C, Molinari E, Malleo G, et al. Early versus late drain removal after standard pancreatic resections: results of a prospective randomized trial. Ann Surg 2010;252:207-14.

42. Beane JD, House MG, Ceppa EP, et al. Variation in Drain Management After Pancreatoduodenectomy: Early Versus Delayed Removal. Ann Surg 2019;269:718-24.

43. Villafane-Ferriol N, Shah RM, Mohammed S, et al. Evidence-Based Management of Drains Following Pancreatic Resection: A Systematic Review. Pancreas 2018;47:12-7. 